
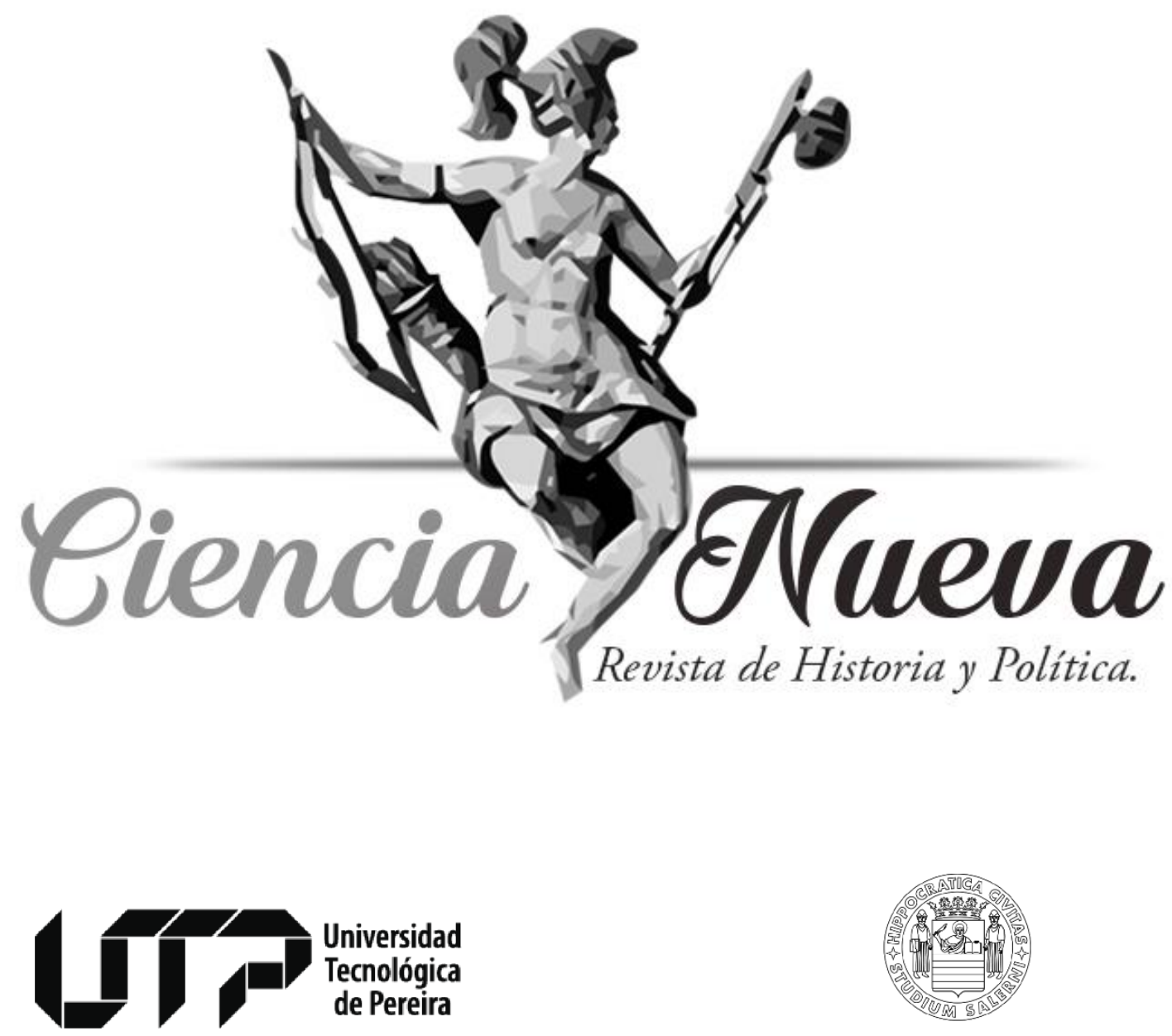

UNIVERSITÀ DEGLI STUDI DI SALERNO

Maestría en Historia

Maestría en Ciencia Política

RESEÑAS

JHON JAIME CORREA RAMÍREZ ET AL., 50/60 UNA HISTORIA COMPARTIDA. INVESTIGACIÓN 50 AÑOS DE RISARALDA Y 60 AÑN DE COMFAMILIAR. MAPA HISTÓRICO CULTURAL (BOGOTÁ: EDITORIAL PLANETA, 2016), 349 PP. JHON JAIME CORREA RAMÍREZ ET AL., 50/60 A SHARED HISTORY. RESEARCH 50 TH ANNIVERSARY OF RISARALDA AND $60^{T H}$ ANNIVERSARY OF COMFAMILIAR. CULTURAL HISTORICAL MAP (BOGOTÁ: EDITORIAL PLANETA, 2016), 349 PP. Gerardo Andrés Zapata Ospina pp. 162-166

Vol. $1 N^{o}$ 2, Julio-Diciembre de 2017 Pereira, Colombia 


\title{
JHON JAIME CORREA RAMÍREZ ET AL., 50/60 UNA HISTORIA COMPARTIDA. INVESTIGACIÓN 50 AÑOS DE RISARALDA Y 60 AÑOS DE COMFAMILIAR. MAPA HISTÓRICO CULTURAL (BOGOTÁ: EDITORIAL PLANETA, 2016), 349 PP.* JHON JAIME CORREA RAMÍREZ ET AL., 50/60 A SHARED HISTORY. RESEARCH 5OTH ANNIVERSARY OF RISARALDA AND 6OTH ANNIVERSARY OF COMFAMILIAR. CULTURAL HISTORICAL MAP (BOGOTÁ: EDITORIAL PLANETA, 2016), 349 PP.
}

\author{
Gerardo Andrés Zapata Ospina** \\ geanzapata@utp.edu.co
}

ORCID: http://orcid.org/0000-0002-5571-6992

\begin{aligned} & \hline Recibido: 30 de marzo de 2017 \\ & Revisado: 30 de abril de 2017 \\ & Aceptado: 09 de junio de 2017 \\ & Publicado: 22 de agosto de 2017 \\ & \hline\end{aligned}

\begin{abstract}
No olvidamos de todos modos, que estos ejercicios, descriptivos en algunos casos, clasificatorios en otros, tratan de aquello que Pierre Bourdieu señala como "luchas por el monopolio del poder de hacer ver y hacer creer, de hacer conocer y de hacer reconocer, de imponer la definición legitima de las divisiones del mundo social y, por ello, de hacer y deshacer grupos: (...) la regio y sus fronteras ( fines) no son más que el trazo muerto del acto de autoridad, consistente en circunscribir el país, el territorio (que se dice también fines) imponer la definición (otro sentido de finis) legitima, conocida y reconocida, de las fronteras y el territorio; en definitiva el principio de división legitima del mundo social"'.
\end{abstract}

Tras los rasgos sensibles del paisaje, tras los escritos en apariencia más fríos y las instituciones en apariencia más distanciadas de quienes las establecieron, la historia quiere captar a los hombres. Quien no lo logre nunca será, en el mejor de los casos, sino un obrero manual de la erudición. El buen historiador se parece al ogro de la leyenda. Ahí donde olfatea carne humana, ahí sabe que está su presa $^{2}$.

uando se decide hacer la historia de un departamento por pequeño que este sea, se emprende de inmediato un reto de magnas dimensiones. Sin embargo, el carácter ambicioso de la propuesta se enfrenta a ciertas barreras que afectan el alcance de los

\footnotetext{
${ }^{*}$ El presente artículo respeta las directrices y normas dispuestas en la Declaración de Ética de Publicación de Ciencia Nueva, Revista de Historia y Política. Esta declaración puede consultarse en la página web de la revista: revistas.utp.edu.co/index.php/historia

** Estudiante de Licenciatura en Etnoeducación y Desarrollo Comunitario e integrante del Semillero de Investigación Formativa en Historia, Cultura Política y Educación, adscrito al grupo de investigación Políticas, Sociabilidades y Representaciones Histórico-Educativas (PSORHE) de la Escuela de Ciencias Sociales de la Facultad de Ciencias de la Educación de la Universidad Tecnológica de Pereira.

${ }^{1}$ Jhon Jaime Correa Ramírez et al. 50/60 Una historia compartida. Investigación 50 años de Risaralda y 60 años de Comfamiliar. Mapa histórico cultural (Bogotá: Planeta, 2016), 24.

${ }^{2}$ Marc Bloch, Apología para la historia o el oficio del historiador (México D.F: Fondo de Cultura Económica, 2001), 57.
} 
objetivos. Para el caso de este libro, se quedan sin resolver algunas importantes propuestas que constituyen el mapa histórico cultural del departamento en el marco de sus 50 años de vida administrativa y política. Por ello, esta reseña intenta mostrar los debates en los que se envuelve el libro y las utilidades de los mapas culturales a la hora de narrar y explicar los 50 años de historia del departamento de Risaralda y los 60 años de Comfamiliar-Risaralda, y el alcance del mismo como investigación histórica regional.

En el marco de la celebración de los 50 años de vida político-administrativa del departamento de Risaralda y los 60 años de creación de la caja de compensación de Risaralda Confamiliar en alianza con la Maestría en Historia, el grupo de investigación en Políticas, Sociabilidades y Representaciones Históricas Educativas de la Universidad Tecnológica de Pereira, lanzan 50/60 Una historia compartida. Dicho trabajo es pionero en su clase, se despliega en la categoría de historia regional y local. Cabe mencionar, que los trabajos realizados anteriormente y que tienen que ver con el estudio histórico del territorio que comprende el departamento de Risaralda, obedecen a monografías de los diferentes municipios y hacen referencia a un periodo anterior a 1967, cuando estos municipios pertenecieron al departamento de Caldas, y continúan después de la separación y luego constitución como el departamento piloto de Colombia. En suma, estas monografías pueden resultar abundantes, pero no poseen el rigor de la ciencia histórica ni de su respectivo profesional para abrir nuevos espacios y enfoques al análisis del pasado, y así dimensionar y proporcionar sentido a la historia. Así, los autores sustentan que pensar la región:

Es como una especie de "vórtice" -con una enorme fuerza centrífuga sobre la que recaen los flujos económicos y demográficos de otras regiones cercanas e incluso lejanas-. Y quizás en esta misma medida, es factible empezar a reconocer una región que si bien ha sido de paso, también ha permitido que se arraiguen personas de muy distintas procedencias. Y en lugar de una identidad cultural única, detenta una condición pluricultural que debe ser objeto de nuevas y más profundas investigaciones, sobre todo cuando se trata de explicar la diversidad y muchos de los conflictos sociales y culturales más recientes. ${ }^{3}$

Los autores consideran que para enfrentar el reto de escribir la historia del departamento de Risaralda y sus procesos culturales, es necesario acudir a una herramienta metodológica que para estos casos resulta adecuada. En consecuencia, los autores explican el procedimiento del instrumento metodológico:

Y en este caso resultan de gran utilidad los mapas culturales, que permiten dar cuentas de otras formas de ver, oír, relacionar, comparar, narrar y explicar una unidad político- administrativa como la del actual departamento de Risaralda. A partir de la consulta bibliográfica, los balances historiográficos, la consulta de archivos, talleres grupales, entrevistas y la juiciosa recopilación etnográfica [...]. ${ }^{4}$

El libro se divide en quince secciones o capítulos que corresponden a cada uno de los catorce municipios que conforman el departamento y uno dedicado a Comfamiliar Risaralda, los cuales, a su vez, se dividen en subsecciones que dan cuenta de varias categorías de análisis:

1. Pasado precolombino.

2. Proceso de colonización.

\footnotetext{
${ }^{3}$ Correa et al., 50/60 Una historia compartida..., 245.

${ }^{4}$ Correa et al., 50/60 Una historia compartida..., 20.
} 
3. Fundación de los poblados y las primeras familias.

4. La violencia política de los 50 y 60.

5. Aspectos y problemáticas contemporáneas.

También en el desarrollo de los capítulos se pone en consideración otras categorías de análisis para procesos sociales más recientes que afectan la región: las migraciones hacia el extranjero, las formas de organización comunitaria de los nativos y el impacto de las nuevas tecnologías de la información en las recientes generaciones.

Con la aplicación de esta metodología, los autores pretendían desmarcarse de la tradicional tendencia de las versiones simplistas de la historia y del continuo cambio en la resignificación de los procesos culturales, de las luchas políticas y sociales, del impacto que generan los cambios económicos generados por las bonanzas cafeteras, el desplome estrepitoso de las mismas a causa de la caída de los precios a fínales de los 80 y finalmente el impacto en la economía regional del narcotráfico.

Se cumple con disciplina el uso de la metodología trazada, además de la aplicación de las categorías de análisis en todos los catorce capítulos, con algunas excepciones en los capítulos escritos para Apia y Santa Rosa de Cabal, en donde el autor irrumpe con una visión diferente a los procesos socio-históricos analizándolos a partir de una visión político-cultural. Así mismo, el estilo de escritura de los capítulos enfocados en estos dos municipios, hace difícil la comprensión de los conceptos que se quieren debatir. También se observa un uso exagerado de las fuentes primarias orales, ya que a partir del testimonio reiterado hay una sola fuente para soportar determinadas afirmaciones sin que se denote una crítica a dichas fuentes. El uso redundante de una sola fuente oral o en su defecto de la misma entrevista colectiva para dar soporte a las afirmaciones expuestas por el autor, van generando ciertas dualidades del investigador en historia, descritas por Cardona Tobón y Martínez Garnica en la investigación de la historia regional y local:

La primera dualidad es la del vocabulario que debe usarse en el relato histórico, es decir, la decisión entre usar los conceptos fabricados en el gabinete de los sociólogos o los conceptos que emanan de las propias fuentes de la investigación histórica. Según la resolución que se tome, elegimos una opción de rigurosidad en el uso del lenguaje del análisis histórico, esto es, entre la preferencia por los lenguajes a la moda o por el lenguaje de los testimonios documentales de las sociedades que nos antecedieron. ${ }^{5}$

Allí se pierde cierto rigor en la ciencia histórica en el momento en que se apoya más en la "memoria" que en los archivos documentales. Por ende es visible la falta de una contrastación de las fuentes de investigación.

En conclusión, un aspecto importante de esta publicación radica en la aparición de un nuevo debate a nivel regional sobre la historia del departamento, el cual queda abierto para superar completamente las visiones historicistas llenas de relatos de corte épico. De forma que las nuevas interpretaciones históricas de tipo profesional sean un punto de partida para una historia con una perspectiva distinta. Este libro como apuesta sugestiva plantea un diálogo entre los mapas culturales y el rigor de la ciencia histórica, utilizando el método etnográfico y la consulta de archivos y fuentes documentales. De esta manera los investigadores en historia obtienen ciertos elementos históricos, suficientes para orientar a los lectores contemporáneos acerca de la representación histórica regional propuesta en el texto, detallando diversos

\footnotetext{
5 Alfredo Cardona Tobón y Armando Martínez Garnica, "La historia regional y la generación de las identidades", Revista Ciencia Nueva 1 (2016): 284.
} 
procesos ocurridos en la construcción de esta región. Lo que permite al mismo tiempo definir ciertas identidades que representan a los risaraldenses, entender las diferentes prácticas clientelistas que incidieron en la separación de Caldas, poner en entredicho el dominio de un imaginario cultural antioqueño y cafetero, superar la invisibilización de los grupos indígenas y afrocolombianos en la historia risaraldense, entre otros.

Queda claro de todas maneras, que aun cuando este trabajo es una investigación pionera que abre un campo de estudio en la historia regional, se trata de una versión resumida que apenas nos permite referenciar con cuidado algunos nuevos elementos culturales del departamento de forma descriptiva. Por ello el ejercicio propuesto de investigación se queda corto, toda vez que dichos elementos histórico culturales no se ponen en un diálogo articulado entre todos los capítulos, sino que por el contrario presentan a cada municipio como un lugar aislado con el resto de poblaciones, presentando así una región llena de islas. 


\section{Bibliografía.}

Bloch, Marc. Apología para la historia o el oficio del historiador. México D.F.: Fondo de Cultura Económica, 2001.

Cardona Tobón, Alfredo y Armando Martínez Garnica. "La historia regional y la generación de las identidades". Revista Ciencia Nueva 1 (2016).

Correa Ramírez, Jhon Jaime, Anderson Paul Gil Pérez, Jhon Anderson Tascón Bedoya, Edwin Mauricio López García y Maribel Valencia López. 50/60 Una historia compartida. Investigación 50 años de Risaralda y 60 años de Comfamiliar. Mapa histórico cultural. Bogotá: Planeta, 2016. 